\title{
Dynamic Stability of an Elastic Rotating System: Shell-Disc-Shaft
}

\author{
P. RUGE, PROF. DR.-ING. and P. SENKER, DR.-ING. \\ Technical University Dresden, Germany
}

(Received 5 August 1996)

\begin{abstract}
This paper presents a methodology and some results on the dynamic stability of an elastic rotating system consisting of one- and twodimensional members. These parts may contain different kinds of unsymmetries: either from mass- or stiffness imperfections or from anisotropic especially hydrodynamic bearings. The equations of motion are formulated using virtual work and an Finite Element approach. Special attention is paid to a kinematically consistent coupling of the elastic shell and disc. The eigenvalue extraction is based upon the method of Lanczos including a modal reduction and a correction process in order to ensure true diagonal system matrices. Some typical results for a shaft-disc-shell system with different bearings and imperfections are presented in detail.
\end{abstract}

Keywords: Dynamic Stability, Elastic Rotorsystems, Lanczos Eigenvalue Solver, Imperfections.

\section{INTRODUCTION}

Rotating elastic systems including thin cylindrical shells, discs and shafts are widely used in mechanical, aeronautical and marine engineering. Modern design of electrical machines, for example, includes thin-walled bell-type rotors preferably made of composite material in multilayered mode. The elasticity of such shells may have a significant influence on the overall dynamic behavior of a system like that shown in Fig. 1. The mechanical and geometric properties of the other parts of the system, too, do effect the stability. From classical one-dimensional studies it is very well known, that imperfections and deviations from any symmetries can cause significant effects on the dynamic stability. This paper presents results for a system with following members:

- Rotor shaft with small deviations compared with the ideal round shape of the cross-section.

- Bell-type thin shell made of homogeneous material, prestressed by the radial acceleration forces with local perturbations; for example caused by a discrete load system with mechanical properties of its own.

- Elastic disc connecting shaft and shell. This disc is prestressed, too, and may have mass and stiffness imperfections.

- Anisotropic, for example hydrodynamic bearings.

An overall analysis of such a coupled system has not been published in literature. Results for only parts of this system are especially given for flexible turbine 


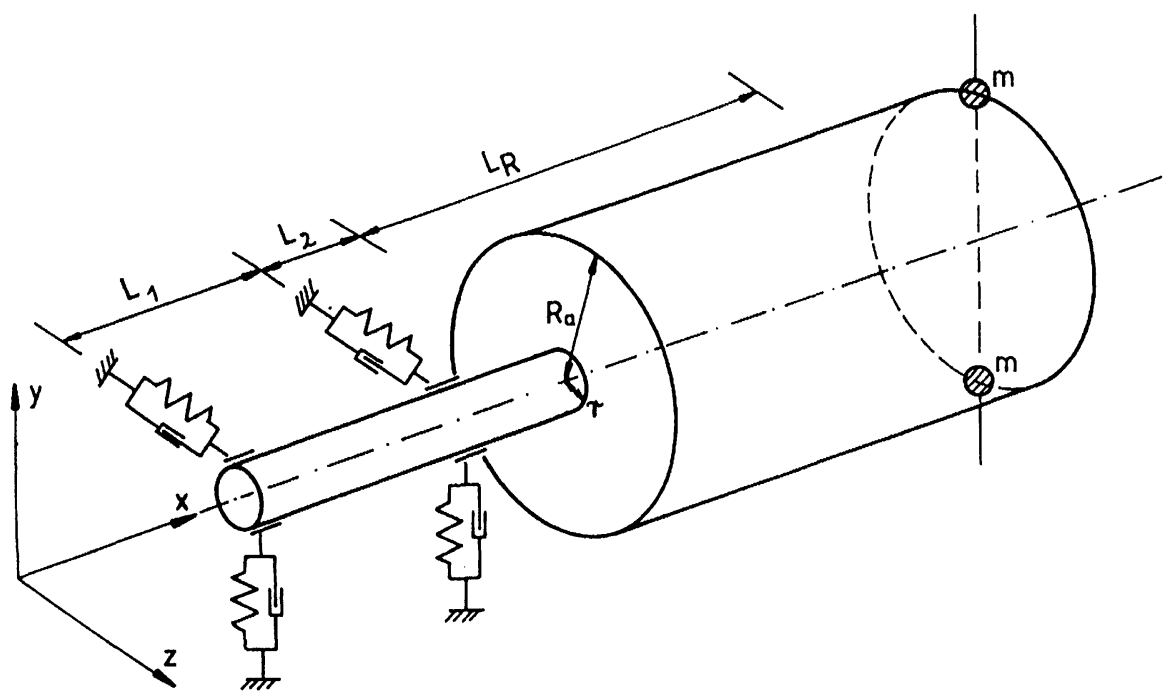

FIGURE 1 Elastic rotating system with hydrodynamic bearings and additional pair of discrete masses

blades and flexible spinning discs in the field of rotordynamics by Sakata et al. [1989], Staudinger [1993], Wauer and Seemann [1989], Steigleder and Krämer [1989], Omprakash and Ramamurti [1990], Yano and Kotera [1991]. Other papers like that by Chen and Bogy [1992] are motivated by computer disc drives in contact with a loading system.

A global result of these papers is that the membran stresses in the rotating members have a significant influence on the stiffness of the system; in structural analysis this effect is called "geometric stiffness". The way to involve this effect into the analysis starts from a nonlinear representation of the strains $\epsilon$ by means of the displacements $\boldsymbol{u}$ and slopes $\boldsymbol{\beta}$.

Rotating thin cylindrical shells of finite length with arbitrary boundary conditions including the geometric stiffness are rarely treated in applied mechanics. Plonski and Ruge [1990] presented some results for spinning shells with mass imperfections and different multilayered composites but with rigid disc and rigid bearings. There are some few papers dealing with finite perfect cylindrical shells by Suzuki et al. [1991], with ring-stiffened shells by Huang and Shu [1992] and dealing with true three-dimensional solid and hollow cylinders; Seemann [1992]. The situation for rotating rings is much easier and there are some profound studies by Clemens and Wauer [1985] and Wauer [1987] which confirm experimental results published by Endo et al. [1984].

Our attempt was to design a numerical tool which allows a true three-dimensional analysis of spinning coupled systems without imposing symmetry or isotropy conditions. We restrict ourselves to the stationary situation with constant angular velocity $\Omega$ and locally linear behavior of the fluid-film, which is nonlinear in general.

Whatever basis we use for describing the state equations we will eventually get a system of linearized differential equations in the time domain with periodic coefficients like $\cos (2 \Omega t), \sin (2 \Omega t)$.

$$
\begin{aligned}
& \boldsymbol{A} \ddot{\boldsymbol{q}}+\boldsymbol{B} \dot{\boldsymbol{q}}+\boldsymbol{C} \boldsymbol{q}=\boldsymbol{r}, \\
& \boldsymbol{A}=\boldsymbol{M}_{2}, \boldsymbol{B}=2 \Omega \boldsymbol{M}_{1}+\boldsymbol{D}_{B}^{0}(\Omega t)+\boldsymbol{D}_{S}, \\
& \boldsymbol{C}=\boldsymbol{K}_{B}(\Omega t)+\boldsymbol{K}_{S}^{L}+\boldsymbol{K}_{S}^{M S}+\Omega \boldsymbol{D}_{B}^{1}(\Omega t)-\Omega^{2} \boldsymbol{M}_{0}, \\
& (. .)_{B}: \text { Bearing; }(. .)_{S}: \text { Structure; }(. .)^{L}: \text { Linear; } \\
& (. .)^{M S}: \text { Membrane Stiffening, } \boldsymbol{r}: \text { radial } \\
& \quad \text { mass-acceleration forces; }
\end{aligned}
$$


$\boldsymbol{M}_{i}:$ Caused by inertia mass; $\boldsymbol{D}^{i}$ : Damping;

$$
K \text { :Stiffness. }
$$

Most of the degrees of freedom $\boldsymbol{q}$ in (1) describe the displacements and slopes of the continuous elastic members which are discritized by a Finite Element approximation. Consequently we decided to use a rotating basis $\boldsymbol{A}$ fixed to the undeformed constantly rotating configuration. Then only some few discrete displacements and slopes of the points where the bearings are fixed to the shaft are associated with periodic coefficients.

\section{KINEMATICS, VIRTUAL WORK}

The virtual work $\delta W$ of the internal elastic forces and the inertia forces is mainly described by the position vector $\overrightarrow{\boldsymbol{x}}_{P}$ which depends on the displacement-vector
$\overrightarrow{\boldsymbol{u}}_{P}$ of an arbitrary point $\mathrm{P}$ in Fig. 2 outside the middle-surface of the shell.

$$
\overrightarrow{\boldsymbol{x}}_{P}=\xi \overrightarrow{\boldsymbol{a}}_{1}+(R+\zeta) \overrightarrow{\boldsymbol{a}}_{3}+\overrightarrow{\boldsymbol{u}}_{P}
$$

The procedures for treating the disc and the shaft are rather similar. The vector $\overrightarrow{\boldsymbol{u}}_{P}$ consists of two typical parts: a translational and a rotational one.

$$
\begin{gathered}
\overrightarrow{\boldsymbol{u}}_{P}=\overrightarrow{\boldsymbol{u}}+\zeta d \overrightarrow{\boldsymbol{a}}_{3}, \quad d \overrightarrow{\boldsymbol{a}}_{3}=\overrightarrow{\boldsymbol{\beta}} \times \overrightarrow{\boldsymbol{a}}_{3} . \\
\hookrightarrow \overrightarrow{\boldsymbol{u}}_{P}=\overrightarrow{\boldsymbol{u}}-\zeta \overrightarrow{\boldsymbol{a}}_{3} \times \overrightarrow{\boldsymbol{\beta}} .
\end{gathered}
$$

The rotational part in (3) results from linearizing the fundamental velocity equation $d \boldsymbol{a} / d t=\dot{\boldsymbol{\beta}} \times \boldsymbol{a}$. The displacements $\overrightarrow{\boldsymbol{u}}$ and slopes $\overrightarrow{\boldsymbol{\beta}}$ are measured with respect to the rotating basis $\boldsymbol{A}$, where $\boldsymbol{u}$ and $\boldsymbol{\beta}$ without vector-sign are the coordinates.

$$
\vec{u}=A u, \quad \vec{\beta}=A \beta .
$$

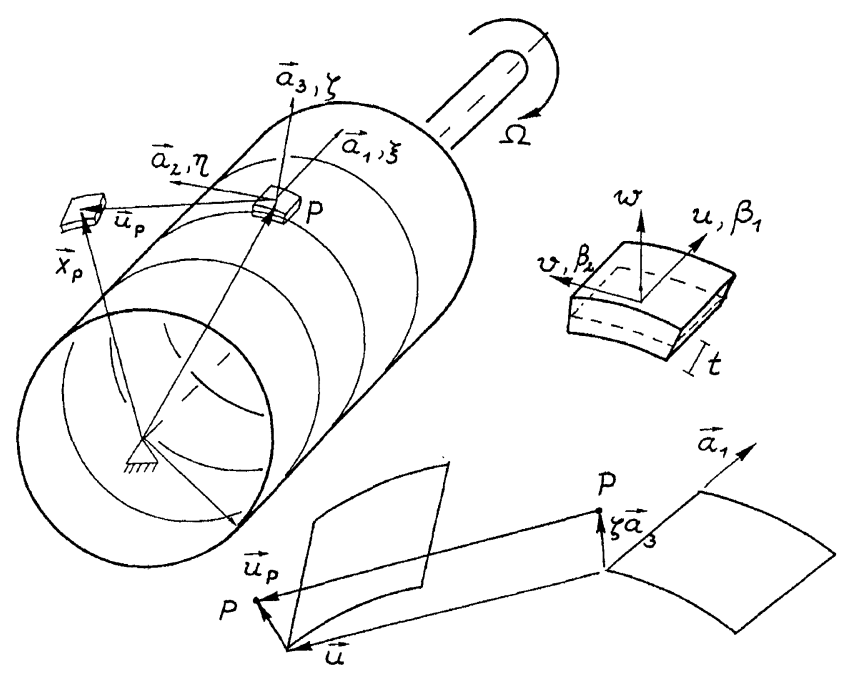

FIGURE 2 Notations, position- and displacement vector of a point $\mathrm{P}$ outside the middle-surface of shell. 


$$
\begin{gathered}
\overrightarrow{\boldsymbol{u}}_{P}=\boldsymbol{A}\left(\boldsymbol{u}-\zeta\left[\begin{array}{l}
0 \\
0 \\
1
\end{array}\right] \times\left[\begin{array}{l}
\beta_{1} \\
\beta_{2} \\
0
\end{array}\right]\right)=\boldsymbol{A}\left(\boldsymbol{u}-\zeta \tilde{\boldsymbol{\imath}}_{3} \boldsymbol{\beta}\right) \\
\boldsymbol{i}_{3}=\left[\begin{array}{l}
0 \\
0 \\
1
\end{array}\right] .
\end{gathered}
$$

The vector-product $\left(\boldsymbol{i}_{3} \times\right)=\tilde{\boldsymbol{i}}_{3}$ is formulated by means of a skew-symmetric or gyroscopic arrangement proposed by Wittenburg [1977].

$$
\begin{gathered}
(\boldsymbol{a} \times)=\tilde{\boldsymbol{a}}=\left[\begin{array}{ccc}
0 & -a_{3} & a_{2} \\
a_{3} & 0 & -a_{1} \\
-a_{2} & a_{1} & 0
\end{array}\right] . \\
\tilde{\boldsymbol{i}}_{3}=\left[\begin{array}{ccc}
0 & -1 & 0 \\
1 & 0 & 0 \\
0 & 0 & 0
\end{array}\right]=\boldsymbol{G}_{3} .
\end{gathered}
$$

The last, rotational, part in (5) is governed by the elastic slopes $\boldsymbol{\beta}$, which are assumed to be independent of the displacements and to be very small: $\sin \beta$ $\approx \beta, \cos \beta \approx 1$. This independent choice of slopes has several advantages:

- Simultaneous representation of translational and rotational inertia.

- Simple continuity conditions with respect to the variational approach; $C^{0}$-continuity only.

- Automatic treatment of shear elasticity.

The virtual displacement $\delta \overrightarrow{\boldsymbol{u}}_{P}$ follows directly from (5),

$$
\delta \overrightarrow{\boldsymbol{u}}_{P}=\boldsymbol{A}\left(\delta \boldsymbol{u}-\zeta \boldsymbol{G}_{3} \delta \boldsymbol{\beta}\right) .
$$

The acceleration $\ddot{\vec{u}}_{P}$ is easy to describe if one uses the fundamental skew symmetric representation,

$$
\dot{\boldsymbol{A}}=\boldsymbol{A} \tilde{\boldsymbol{\omega}}=\boldsymbol{A} \Omega \tilde{\boldsymbol{\imath}}_{1}=\boldsymbol{A} \Omega \boldsymbol{G}_{1} \cdot \tilde{\boldsymbol{\iota}}_{1}=\boldsymbol{G}_{1}=\left[\begin{array}{ccc}
0 & 0 & 0 \\
0 & 0 & -1 \\
0 & 1 & 0
\end{array}\right],
$$

for $\dot{A}$ with the local angular velocity $\boldsymbol{\omega}$, which simplifies to $\omega=\Omega \boldsymbol{a}_{1}$ in our specific situation.

$$
\begin{aligned}
\ddot{\vec{u}}_{P}=\boldsymbol{A}(\ddot{\boldsymbol{u}} & \left.+2 \Omega \boldsymbol{G}_{1} \dot{\boldsymbol{u}}+\Omega^{2} \boldsymbol{G}_{1}^{2} \boldsymbol{u}\right)+\boldsymbol{A} \zeta \boldsymbol{G}_{3}(\ddot{\boldsymbol{\beta}} \\
& \left.+2 \Omega \boldsymbol{G}_{1} \dot{\boldsymbol{\beta}}+\Omega^{2} \boldsymbol{G}_{1}^{2} \boldsymbol{\beta}\right) .
\end{aligned}
$$

The virtual displacements from (7) and the acceleration from (9) are taken to describe the virtual work of the inertia forces. If the middle-surface with $\zeta=0$ is the geometric center in direction $\overrightarrow{\boldsymbol{a}}_{3}$ of the shell, that means $\int \zeta d \zeta=0$, the rotational and translational inertia parts are decoupled; $\rho$ is the mass density, $t$ the thickness of the shell.

$$
\begin{aligned}
& \delta W= \int \delta \vec{u}_{P} \cdot \ddot{\vec{x}}_{P} \rho d \xi d \eta d \zeta \\
& \delta W=\int \delta \boldsymbol{u}^{T}\left(\ddot{u}+2 \Omega \boldsymbol{G}_{1} \dot{\boldsymbol{u}}+\Omega^{2} \boldsymbol{G}_{1}^{2} \boldsymbol{u}\right) \rho t d \xi d \eta \\
& \quad-\int \delta \boldsymbol{u}^{T} \Omega^{2} R \boldsymbol{i}_{3} \rho t d \xi d \eta \\
&+\int \delta \boldsymbol{\beta}^{T} \boldsymbol{G}_{3}^{2}\left(\ddot{\beta}+2 \Omega \boldsymbol{G}_{1} \beta+\Omega^{2} \boldsymbol{G}_{1}^{2} \boldsymbol{\beta}\right) \rho \frac{t^{3}}{12} d \xi d \eta
\end{aligned}
$$

The virtual elastic work $\delta U$, here presented in detail for the cylindrical shell, consists of three parts $U_{B}$, $U_{M}, U_{S}$ caused by bending moments $\boldsymbol{m}$, inplane- or membrane forces $\boldsymbol{n}$ and shear forces $\boldsymbol{q}$.

$$
\begin{gathered}
U_{B}=\frac{1}{2} \int \boldsymbol{\kappa}^{T} B \boldsymbol{E} \boldsymbol{\kappa} d \xi d \eta \text { with } \boldsymbol{m}=B \boldsymbol{E} \boldsymbol{\kappa}, \\
U_{M}=\frac{1}{2} \int \boldsymbol{\epsilon}^{T} D \boldsymbol{E} \boldsymbol{\epsilon} d \xi d \eta \text { with } \boldsymbol{n}=D \boldsymbol{E} \boldsymbol{\epsilon}, \\
U_{S}=\frac{1}{2} \int \gamma^{T} G \gamma d \xi d \eta \text { with } \boldsymbol{q}=G t \boldsymbol{\gamma}, \\
\boldsymbol{E}=\left[\begin{array}{lll}
1 & v & 0 \\
v & 1 & 0 \\
0 & 0 & \frac{1-v}{2}
\end{array}\right], D=\frac{E t}{1-v^{2}}, B=D \frac{t^{2}}{12} .
\end{gathered}
$$

The strains and curvatures $\epsilon, \gamma, \kappa$ are functions of the displacements $\boldsymbol{u}$ and slopes $\boldsymbol{\beta}$ and can be found in textbooks. 


$$
\begin{gathered}
\boldsymbol{\epsilon}=\left(\begin{array}{c}
u,,_{\xi} \\
v_{,_{\eta}}+w / R \\
u,_{\eta}+v,,_{\xi}
\end{array}\right) \\
+\frac{1}{2}\left(\begin{array}{c}
u,_{\xi}^{2}+v,_{\xi}^{2}+w,_{\xi}^{2} \\
\left(v_{,_{\eta}}+w / R\right)^{2}+u,_{\eta}^{2}+\left(w,_{\eta}-v / R\right)^{2} \\
2\left[\left(v_{,_{\eta}}+w / R\right) v,_{\xi}+u, \xi,_{\eta}+\left(w,_{\eta}-v / R\right) w, \xi\right.
\end{array}\right) .
\end{gathered}
$$

$$
\begin{gathered}
\boldsymbol{\kappa}=\left(\begin{array}{c}
-\beta_{2, \xi} \\
\beta_{1, \eta}+v_{,_{\eta}} / R+w / R^{2} \\
\beta_{1, \xi}-\beta_{2, \eta}+v, \xi
\end{array}\right), \\
\gamma=\left(\begin{array}{c}
w,{ }_{, \xi}-\beta_{2} \\
w m_{\eta}-v / R+\beta_{1}
\end{array}\right) .
\end{gathered}
$$

The nonlinear part in (13) must be incorporated into the analysis in order to describe the geometric stiffening. Simplified versions for this nonlinear part are known, for example given by Donnel and Marguerre. However, there are regions of distinct parameters, where results based on Donnel-Marguerre's approximation differ too much from the better ones based on (13). Finally, the sum of the virtual works is taken to describe the state equations of the problem under consideration.

$\delta W+\delta U=\int[\delta \boldsymbol{u} \delta \boldsymbol{\beta}]^{T} \boldsymbol{d}(\boldsymbol{u}, \boldsymbol{\beta}) d \xi d \eta=0$

The column $\boldsymbol{d}$ in (15) depends on the accelerations (9) and strains (13), (14) which are functions of the slopes and displacements.

\section{FINITE ELEMENT APPROACH}

An independent treatment of displacements and slopes as introduced in section 2 allows for shear deformations and rotational inertia effects and has to ensure nothing but continuity with respect to $\boldsymbol{u}$ and $\boldsymbol{\beta}$ along adjacent elements. This formulation and procedure was introduced by Reissner and Mindlin.

A further aspect with direct influence on the number of degrees of freedom is the FEM-net which must be able to describe the essential modes with respect to the dynamic behavior. The necessity to assign a rather fine net results from the wellknown effect in linear analysis that cylindrical shells tend to vibrate in first-order modes with several nodes in circumferential direction. It has been found that a FEM-net with 40 elements in circumferential direction and 4 elements in direction of the shell-axis gives enough freedom to the shell. Adding the degrees of freedom for the disc and the shaft gives a total number of round about $n=6000$ DOFs incorporated in the column $\boldsymbol{q}$ in (1).

Such an approximation is based upon an 8-node element with polynomials in both directions of the shell surface indicated by the coordinates $\xi$ and $\eta$. Alternative interpolations use polynomials in direction of the shell axis, for example a linear interpolation, and a kind of Fourier-expansion in circumferential direction.

$u(\xi, \eta)=\left(1-\frac{\xi}{h}\right) \boldsymbol{t}^{T} \boldsymbol{a}_{0}+\frac{\xi}{h} \boldsymbol{t}^{T} \boldsymbol{a}_{1}, \quad 0 \leq \xi \leq h$,

$\boldsymbol{t}^{T}=[1 \cos \varphi \cos 2 \varphi \ldots \sin \varphi \sin 2 \varphi \ldots], \varphi=\eta / R$,

$$
0 \leq \varphi \leq 2 \pi
$$

$\boldsymbol{a}^{T}=\left[a_{0} a_{1} a_{2} \ldots b_{1} b_{2} \ldots\right]$.

Such an approach is normalized with respect to the overall-quantities of a total ring around the shell, not with respect to discrete nodes. Adding a discrete local mass imperfection in a special point at such a ring causes a distribution upon the whole element-massmatrix. In other words, such a discrete mass (or stiffness) destroys the diagonal structure of the element matrices usually guaranteed by the orthogonality of the trigonometric functions in circumferential direction. There is not doubt about the global flexibility of the classical two-dimensional polynomial approximation. So we decided to adopt this approach throughout the whole analysis. The result of this procedure is the matrix equation (1) with matrices which are substructured in a very significant manner as is shown by 
Senker [1993] in detail. The strategy to solve this equation consists of three main steps:

1. Treating the quasi-static situation with $\boldsymbol{q}=\boldsymbol{q}_{0}, \dot{\boldsymbol{q}}_{0}$ $=0$ and taking into account constant parts ()$*$ of the matrices only.

$$
\left(\boldsymbol{K}_{\mathrm{S}}^{\mathrm{L}}+\boldsymbol{K}_{\mathrm{B}}^{*}+\Omega \boldsymbol{D}_{\mathrm{B}}^{1^{*}}-\Omega^{2} \boldsymbol{M}_{0}\right) \boldsymbol{q}_{0}=\Omega^{2} \mathrm{R} \boldsymbol{f} .
$$

The right hand column $f$ represents the radial acceleration forces caused by the given rotation. The product $\Omega^{2} R$ is separated in order to make a comparison with the corresponding part $\Omega^{2} \boldsymbol{M}_{0} \boldsymbol{q}_{0}$ from the left side in (18). The nodal quantities in $\boldsymbol{q}$ are slopes and displacements caused by elasticity and of course their magnitude is less than one per thousand of the radius $R$ of the shell; the massfactors $\boldsymbol{f}$ and $\boldsymbol{M}$ do not alter this fact:

$$
R \boldsymbol{f} \gg \boldsymbol{M q _ { 0 }} .
$$

The part $\Omega D_{B}^{1^{*}}$, too, has been proved to be without numerical influence and finally the quasi static solution $\boldsymbol{q}_{0}$ can be calculated from a simplified equation,

$$
\left(\boldsymbol{K}_{S}^{L}+\boldsymbol{K}_{B}^{*}\right) \boldsymbol{q}_{0}=\Omega^{2} R \boldsymbol{f},
$$

with a constant coefficient matrix, which is not affected by the angular velocity $\Omega$.

2. The nodal quantities $\boldsymbol{q}_{0}$ from (20) are supplemented by linear time-dependent variations $\boldsymbol{q}^{+}$,

$$
\boldsymbol{q}=\boldsymbol{q}_{0}+\boldsymbol{q}^{+}(t)
$$

to study the local time behavior of the static solution $\boldsymbol{q}_{0}$. Inserting (21) into the virtual work of the inertia and elastic forces and omitting higher than linear terms results in a linear homogeneous differential equation in the time domain with the geometric stiffness part $\boldsymbol{K}_{S}^{M S}$ which is a function of $\boldsymbol{q}_{0}$ and appears automatically.

$$
\begin{gathered}
\boldsymbol{M}_{2} \ddot{q}^{+}+\left[2 \Omega \boldsymbol{M}_{1}+\boldsymbol{D}_{B}^{0}(\Omega t)+\boldsymbol{D}_{S}\right] \dot{q}^{+} \\
+\left[\boldsymbol{K}_{B}(\Omega t)+\boldsymbol{K}_{S}^{L}+\boldsymbol{K}_{S}^{M S}+\Omega \boldsymbol{D}_{B}^{1}(\Omega t)\right. \\
\left.-\Omega^{2} \boldsymbol{M}_{0}\right] \boldsymbol{q}^{+}=\mathbf{0} .
\end{gathered}
$$

In order to treat this equation by standard algo- rithms it has to be rewritten as first order system,

$$
\begin{gathered}
\boldsymbol{H} \dot{\boldsymbol{p}}+\boldsymbol{G} \boldsymbol{p}=\mathbf{0}, \\
\boldsymbol{H}=\left[\begin{array}{cc}
\boldsymbol{O} & \boldsymbol{I} \\
\boldsymbol{M}_{2} & 2 \Omega \boldsymbol{M}_{1}+\boldsymbol{D}_{B}^{0}+\boldsymbol{D}_{S}
\end{array}\right], \boldsymbol{p}=\left[\begin{array}{l}
\dot{q}^{+} \\
\boldsymbol{q}^{+}
\end{array}\right], \\
\boldsymbol{G}=\left[\begin{array}{cc}
-\boldsymbol{I} & \boldsymbol{O} \\
\boldsymbol{O} & \boldsymbol{K}_{B}+\boldsymbol{K}_{S}^{L}+\boldsymbol{K}_{S}^{M S}+\Omega \boldsymbol{D}_{B}^{1}-\Omega^{2} \boldsymbol{M}_{0}
\end{array}\right],
\end{gathered}
$$

with unsymmetric matrices $\boldsymbol{H}$ and $\boldsymbol{G}$ in general.

3. The time behavior of the variations $\boldsymbol{q}^{+}$is given by an exponential solution if the matrices involved are constant in time. Otherwise, if some of the matrices are periodic, an additional trigonometric summation must be incorporated as is well known from Hill's method.

$$
\boldsymbol{q}^{+}=\boldsymbol{a} \exp (\lambda t) \quad \text { if matrices are constant. }
$$

$$
\begin{aligned}
\boldsymbol{q}^{+}= & {\left[\boldsymbol{a}_{0}+\boldsymbol{a}_{1} \cos \Omega t+\ldots+\boldsymbol{b}_{1} \sin \Omega t+\ldots\right] \times } \\
& \times \exp (\lambda t) \text { if matrices are } \Omega \text {-periodic. }
\end{aligned}
$$

Or, if one uses Floquet's approach, the Floquet transition matrix $\boldsymbol{F}$ for the first order system (23) must be calculated numerically by stepwise integration along a period $T=2 \pi / \Omega$,

$$
\boldsymbol{p}_{1}=\boldsymbol{F} \boldsymbol{p}_{0}, \boldsymbol{p}_{0}=\boldsymbol{p}(t=0), \boldsymbol{p}_{1}=\boldsymbol{p}(t=T) .
$$

This finite difference equation is solved by $\boldsymbol{p}_{1}=$ $\phi \boldsymbol{p}_{0}$. The eigenvalues $\phi$ from the eigenvalue problem $\boldsymbol{F} \boldsymbol{p}_{0}=\phi \boldsymbol{p}_{0}$ indicate the stability behavior.

So far the steps which have to be done towards a time dependent solution $\boldsymbol{q}=\boldsymbol{q}_{0}+\boldsymbol{q}^{+}(t)$ within a local region around the static situation $\boldsymbol{q}_{0}$. The essential part of this solution is hidden in the eigenvalues $\lambda$ or $\phi$ respectively. The local dynamic stability of the rotating system under consideration is guaranteed if and only if no real part $\delta$ of any of the 2 n eigenvalues $\lambda$ in (24), (25) is greater than zero,

$$
\begin{aligned}
& \qquad \lambda_{i}=\delta_{i}+j \omega_{i}, j^{2}=-1, \\
& \text { Stability iff } \quad \delta_{i} \leq 0, i=1 \ldots 2 n ;
\end{aligned}
$$

or if the modulus of no Floquet-eigenvalue $\phi$ exceeds 1. 
Stability if no $\quad\left|\phi_{i}\right|>1, \quad i=1 \ldots 2 n$.

A typical problem arising from coupling disc and shell must be mentioned in order to treat the rotor system in a proper manner from the mechanical point of view. The FEM-representation consists of 3 nodal displacements but only 2 nodal slopes. With regard to the shell these slopes are $\beta_{1}, \beta_{2}$ along $\overrightarrow{\boldsymbol{a}}_{1}$ and $\overrightarrow{\boldsymbol{a}}_{2}$ as is shown in Fig. 3. For the disc these slopes are $\beta_{2}$ and $\beta_{3}$.

$$
\begin{aligned}
& \text { Shell: } \overrightarrow{\boldsymbol{\beta}}_{\text {Shell }}=\beta_{1} \overrightarrow{\boldsymbol{a}}_{1}+\beta_{2} \overrightarrow{\boldsymbol{a}}_{2}, \\
& \text { Disc: } \overrightarrow{\boldsymbol{\beta}}_{\text {Disc }}=\beta_{2} \overrightarrow{\boldsymbol{a}}_{2}+\beta_{3} \overrightarrow{\boldsymbol{a}}_{3} .
\end{aligned}
$$

The variational foundation of the virtual work representation demands for continuity with regard to displacements (there is no problem at all) and slopes. The slopes $\beta_{1}$ from the shell and $\beta_{3}$ from the disc, however, miss their counterparts from the opposite structure. A mathematically consistent way of treating this situation was described by Kebari and Cassell [1991, 1992]. They introduced an artificial rotation, $\beta_{3}$ for the shell,

$$
\text { Shell: } \beta_{3}=\frac{1}{2}\left(v,,_{\xi}-u,,_{\eta}\right)
$$

and $\beta_{2}$ for the disc in a similar manner. By this the momentum equilibrium normal to the middle-surface is not satisfied a priori but is incorporated in a weak sense into the linear membran part of the virtual work, here shown for the shell only.

$$
\begin{gathered}
\delta \tilde{U}_{M}=D t \int \delta \tilde{\epsilon}_{M}^{T} \tilde{E} \tilde{\epsilon}_{M} d \xi d \eta \\
\tilde{\epsilon}_{M}=\left[\begin{array}{c}
u,_{\xi} \\
v_{\eta}+w / R \\
v,_{\xi}-\beta_{3} \\
u_{\eta}+\beta_{3}
\end{array}\right], \tilde{E}=\left[\begin{array}{cccc}
1 & v & 0 & 0 \\
v & 1 & 0 & 0 \\
0 & 0 & 0 & 1-v \\
0 & 0 & 1-v & 0
\end{array}\right] .
\end{gathered}
$$

Extensive numerical experiments, however, realized by Senker [1993] produced optimal results by using the classical representation with $\beta_{3} \equiv 0$ together with the following slope continuity conditions between disc and shell:

$$
\begin{array}{ll}
\beta_{2(\text { Shell })}= & \beta_{2(\text { Disc })} \\
\text { and } & \beta_{1(\text { Shell })}, \beta_{3(\text { Disc })} \text { independent and uncoupled } \\
\text { or } & \beta_{3(\text { Disc })} \text { free and } \beta_{1(\text { Shell })}=0 .
\end{array}
$$

\section{NUMERICAL ANALYSIS}

Keeping in mind what has to be done to analyse the homogeneous system $\boldsymbol{H} \dot{\boldsymbol{p}}+\boldsymbol{G} \boldsymbol{p}=\boldsymbol{o}$ in (23) of double order $2 \mathrm{n} \approx 12000$, either with constant or $\Omega$-periodic

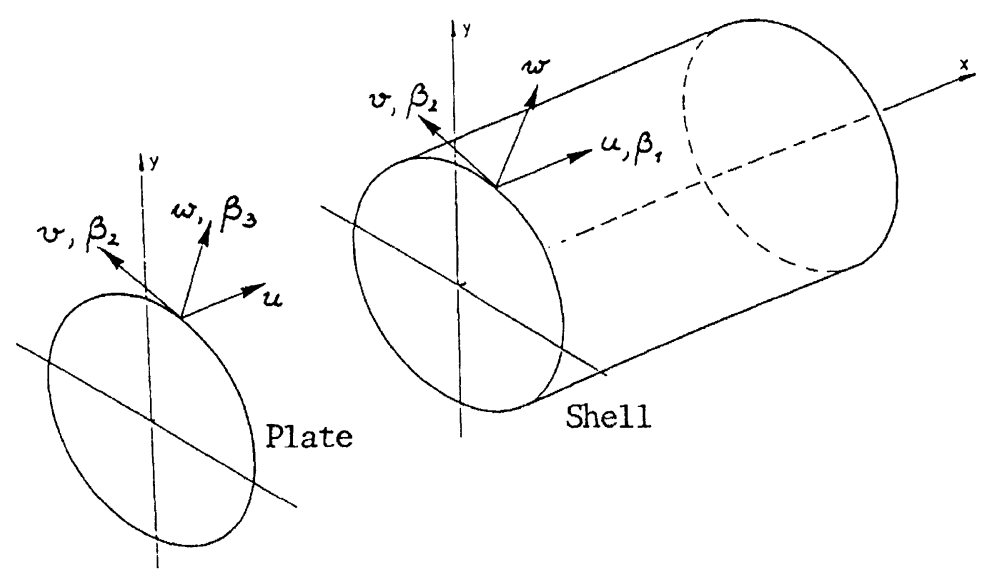

FIGURE 3 Different elastic slopes at the interface of disc and shell. 
matrices, it becomes clear, that the order of the system must be reduced significantly. Even if there are no $\Omega$-periodic parts in $\boldsymbol{H}$ and $\boldsymbol{G}$ these matrices still contain the angular velocity $\Omega$ as a constant parameter and it is necessary to solve the corresponding algebraic eigenvalue-problem

$$
(\lambda \boldsymbol{H}+\boldsymbol{G}) \boldsymbol{z}=\mathbf{o}, \boldsymbol{p}=\boldsymbol{z} \exp (\lambda t)
$$

for each distinct value $\Omega$ separately. Nevertheless, if we restrict the analysis to the first, perhaps 100 eigenvalues, out of the total number or not, this way is far from being competitive. An economic strategy to get satisfying results starts from an auxiliary problem out of the original one (22),

$$
\boldsymbol{M}_{2} \ddot{\boldsymbol{q}}^{+}+\boldsymbol{K} \boldsymbol{q}^{+}=\mathbf{o}, \boldsymbol{K}=\overline{\boldsymbol{K}}_{B}+\boldsymbol{K}_{S}^{L}, \overline{\boldsymbol{K}}=\frac{1}{2}\left(\boldsymbol{K}_{B}+\boldsymbol{K}_{B}^{T}\right),
$$

with symmetric matrices, even if the bearing stiffness is not symmetric in the true situation. A highly efficient method to calculate the lower spectrum of the symmetric problem (34) was described by Lanczos: The original pair $\boldsymbol{M}_{2}, \boldsymbol{K}$ of order $\mathrm{n}$ is transformed into a pair $\boldsymbol{T}, \boldsymbol{I}$ of any order $\mathrm{r}$ less than $\mathrm{n}$ with a tridiagonal matrix $\boldsymbol{T}$.

OriginalPair: $\alpha \boldsymbol{M}_{2} \boldsymbol{y}=\boldsymbol{K} \boldsymbol{y}$.

Transformation: $\boldsymbol{y}=\left[\begin{array}{c}y_{1} \\ \vdots \\ y_{n}\end{array}\right]=\boldsymbol{L} \boldsymbol{z}, \boldsymbol{z}=\left[\begin{array}{c}z_{1} \\ \vdots \\ z_{r}\end{array}\right], r \ll n$,

Resulting Pair: $\alpha \boldsymbol{T} z=\boldsymbol{I}_{r} z, \boldsymbol{L}^{T} \boldsymbol{M}_{2} \boldsymbol{L}=\boldsymbol{T}, \boldsymbol{L}^{T}$ $K L=I_{r}$.

The modal basis

$$
\boldsymbol{Z}=\left[z_{1} \ldots z_{r}\right]
$$

of the auxiliary problem (35) is used to reduce the original complete homogeneous problem (22). It must be kept in mind, however, that the basis $\boldsymbol{Z}$ offers but an approximation,

$$
\boldsymbol{Y}_{r}=\left[\boldsymbol{y}_{1} \ldots \boldsymbol{y}_{r}\right]=\boldsymbol{L} \boldsymbol{Z}_{r},
$$

for the basis $\boldsymbol{Y}_{r}$ of the complete system. This can be seen from the products,

$$
\begin{gathered}
\boldsymbol{Y}_{r}^{T} \boldsymbol{K} \boldsymbol{Y}_{r}=\boldsymbol{E}_{K} \neq \operatorname{diag}_{K}, \\
\boldsymbol{Y}_{r}^{T} \boldsymbol{M}_{2} \boldsymbol{Y}_{r}=\boldsymbol{E}_{M} \neq \operatorname{diag}_{M},
\end{gathered}
$$

which are not purely diagonal as they should be. These errors have been proved to falsify the stability regions of the rotating system in a more or less significant manner. A simple correction, however, starting from a symmetric Cholesky decomposition of the error-matrix $\boldsymbol{E}_{M}=\boldsymbol{S} \boldsymbol{S}^{T}$ allows the calculation of an improved modal basis $\tilde{\boldsymbol{Y}}_{r}$ instead of $\boldsymbol{Y}_{r}$ by forward substitution:

$$
S \tilde{\boldsymbol{Y}}_{r}^{T}=\boldsymbol{Y}_{r}^{T} \rightarrow \tilde{\boldsymbol{Y}}_{r}
$$

Indeed, this basis $\tilde{\boldsymbol{Y}}_{r}$ gives true orthonormality with respect to $\boldsymbol{M}_{2}$,

$$
\tilde{\boldsymbol{Y}}_{r}^{T} \boldsymbol{M}_{2} \tilde{\boldsymbol{Y}}_{r}=\boldsymbol{I}
$$

and no more errors concerning regions of stability have been observed.

\section{EXAMPLES AND CONCLUSION}

Some typical results for a shaft-disc-shell system already shown in Fig. 1 are presented in detail. The angular velocity $\Omega$ is assumed to be constant and the dimensions and material data is choosen as follows:

- Geometry

- Shell: $\mathrm{R}=60 \mathrm{~mm}, \mathrm{t}=0.6 \mathrm{~mm}, \mathrm{l}_{R}=300 \mathrm{~mm}$.

- Disc: $\mathrm{t}=15 \mathrm{~mm}, \mathrm{R}=60 \mathrm{~mm}$.

- Shaft: $1_{1}=220 \mathrm{~mm}, \mathrm{l}_{2}=30 \mathrm{~mm}, \mathrm{r}=15 \mathrm{~mm}$.

- Material

$$
\begin{gathered}
\mathrm{E}=2.0610^{5} \mathrm{~N} / \mathrm{mm}^{2}, \\
\nu=0.3, \rho=7.810^{-9} \mathrm{Ns}^{2} / \mathrm{mm}^{4} .
\end{gathered}
$$



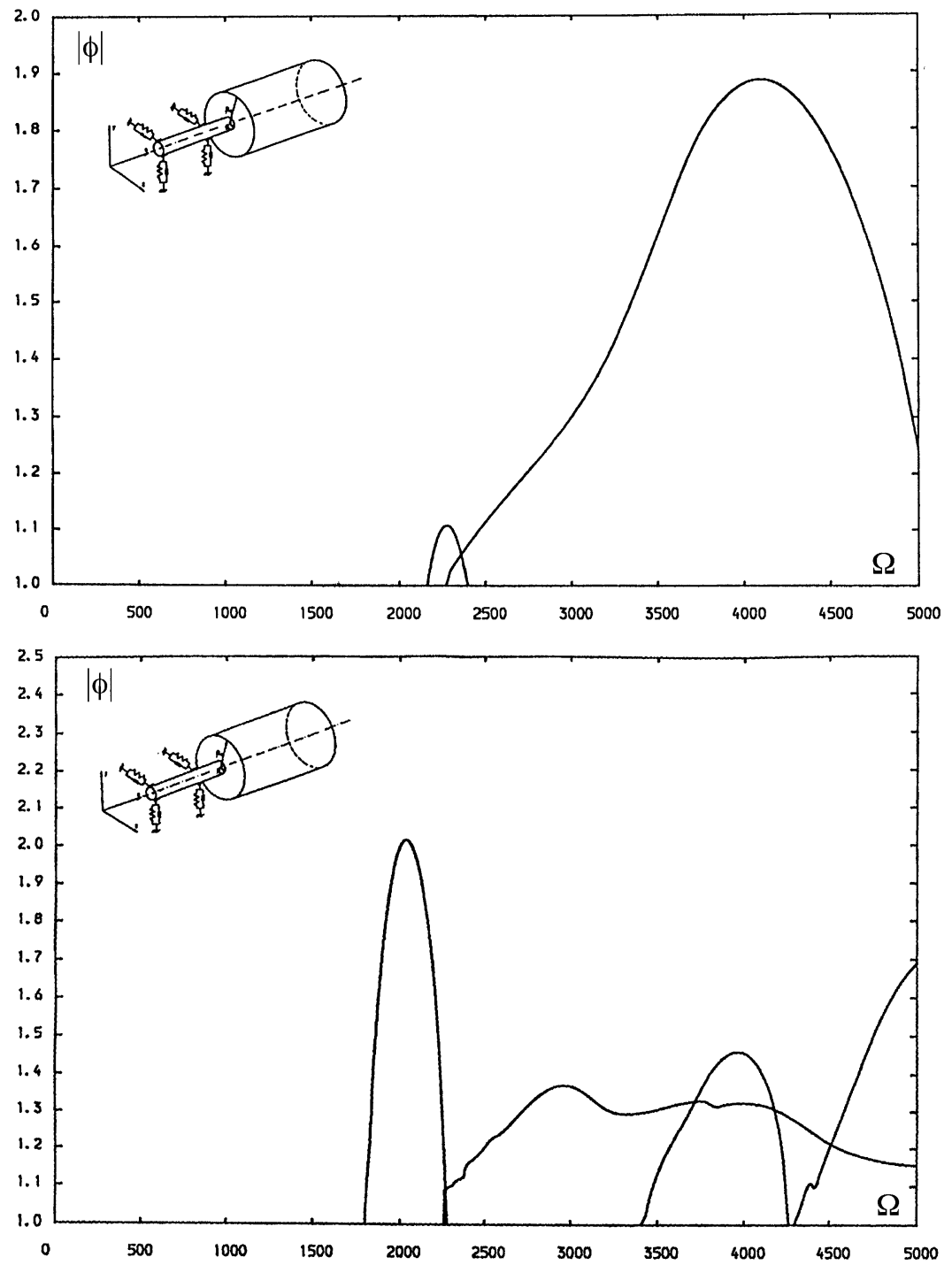

b)

FIGURE 4 Critical modulus $|\phi|$ of Floquet eigenvalues versus angular velocity $\Omega$. a) 2-pads bearings with rigid shell. b) 2-pads bearings with elastic shell. (continued)

The whole elastic system is modelled by $n=6279$ degrees of freedom. By modal reduction however, as was described in chapter 4 , this amount was reduced to $r=60$ remaining DOF's without influence concerning the stability behavior within the lower frequency range. The numerical effort in calculating the eigenvalues $\lambda$ or $\phi$ was not essentially different comparing Hill's and Floquet's method. The results were found to be rather identical. Further informations and a lot of numerical details are presented by Senker [1993].
It is worth mentioning that there may be significant differences between the stability regions of a system either with rigid or with elastic shell. Fig. 4 shows these differences; first for 2-pads bearings in Fig. 4a, b and then for 4-pads bearings in Fig. 4c, d: The elasticity of the shell causes additional regions of instability and decreases the beginning of these regions. The method choosen to examine instability is Floquet's one. Obviously, different types of hydrodynamic bearings can cause rather different results. 
c)

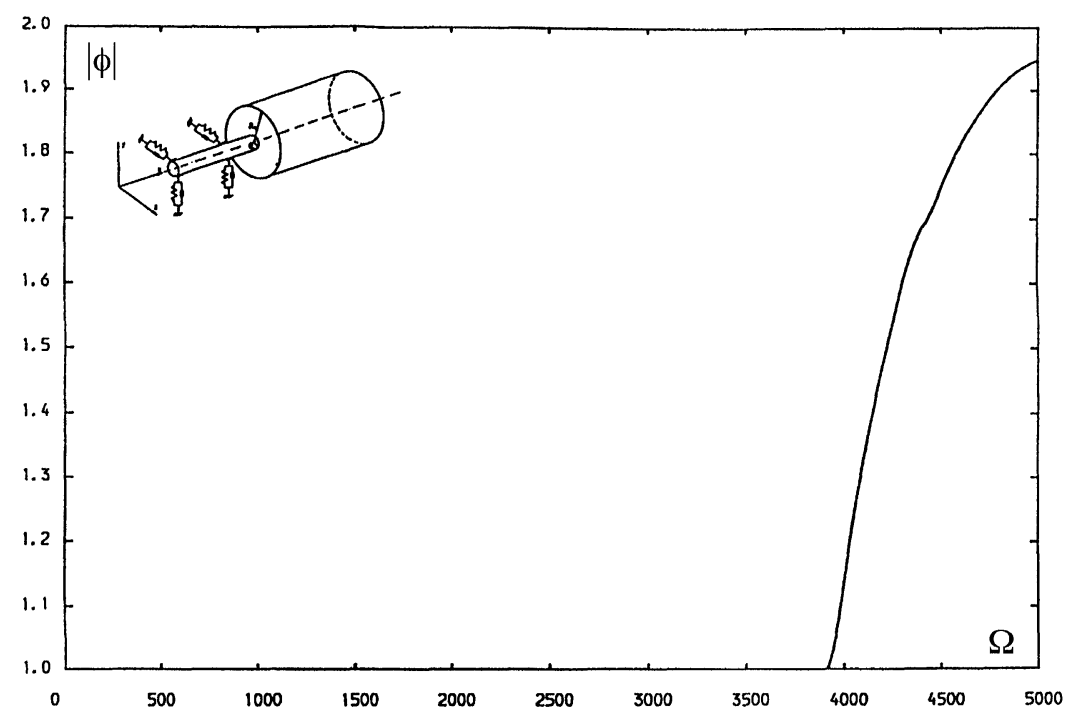

d)

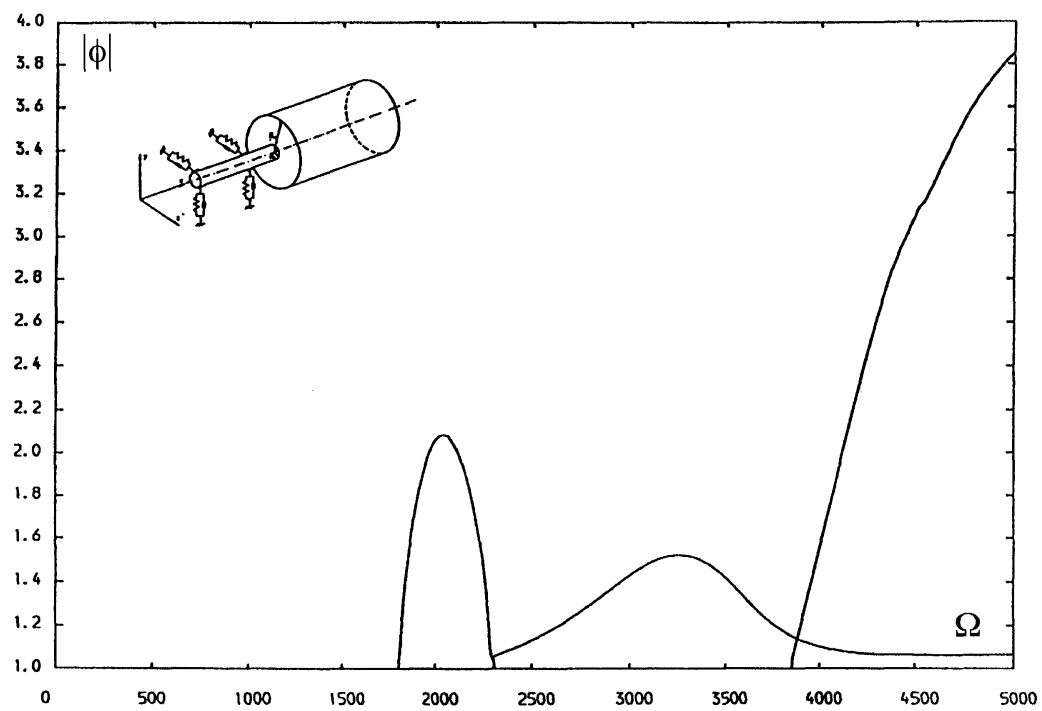

FIGURE 4 (continued) c) 4-pads bearings with rigid shell. d) 4-pads bearings with elastic shell. (continued)

The same system, this time with elasticity for each of the three parts but with an oval shaft indicated by

$$
\left|\frac{c_{\eta}-c_{\zeta}}{c_{\eta}+c_{\zeta}}\right|=1.8, \quad c_{\eta}+c_{\zeta}=10^{8} \mathrm{~N} / \mathrm{m},
$$

gives results in Fig. 4e which are not too different from the situation in Fig. $4 \mathrm{~d}$ for a round shaft. Finally a pair of discrete point-masses is added at the free end of the shell in a symmetrical manner. These two masses together are 3 percent of the total shell mass. Once more we found similar curves in Fig. $4 \mathrm{f}$ as in the previous situations but this time with an additional heavily increasing region of instability which indicates global instability.

These results are only some few out of a great amount which has been accomplished by the second author. To come to a conclusion, we can say that we have established a numerical tool suitable for dealing with the dynamic stability of rotating elastic systems including twodimensional members like shells and discs even with different kinds of unsymmetry. The eigenvalue extraction is based upon the method of Lanczos. It must be emphasized however, that this 
e)

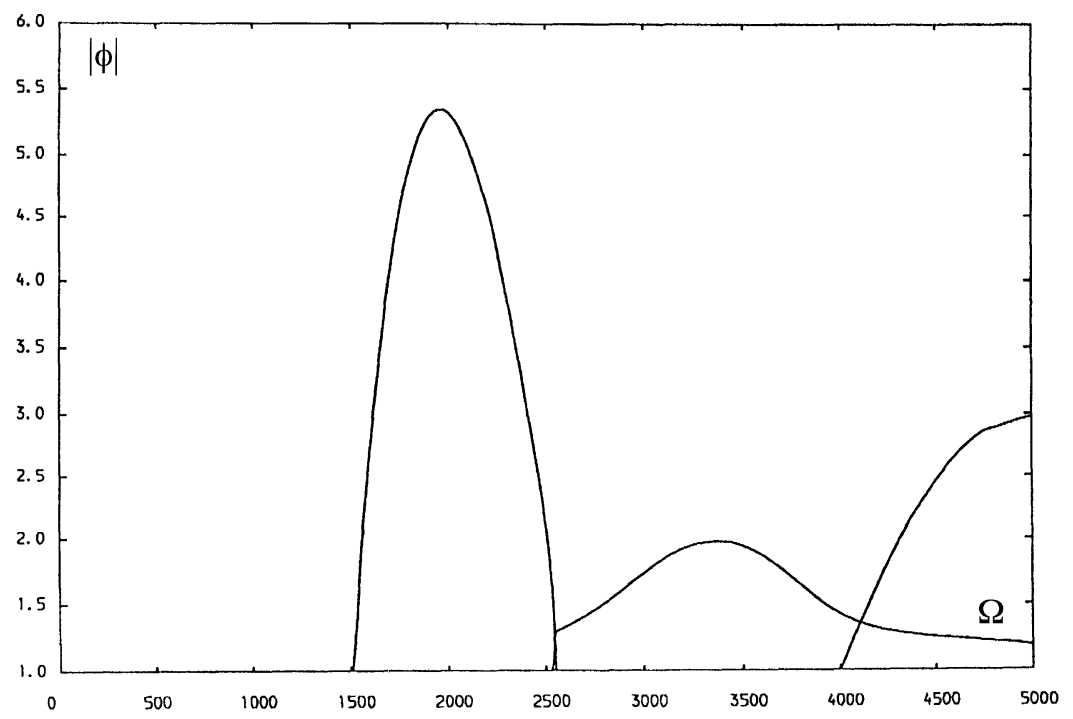

f)

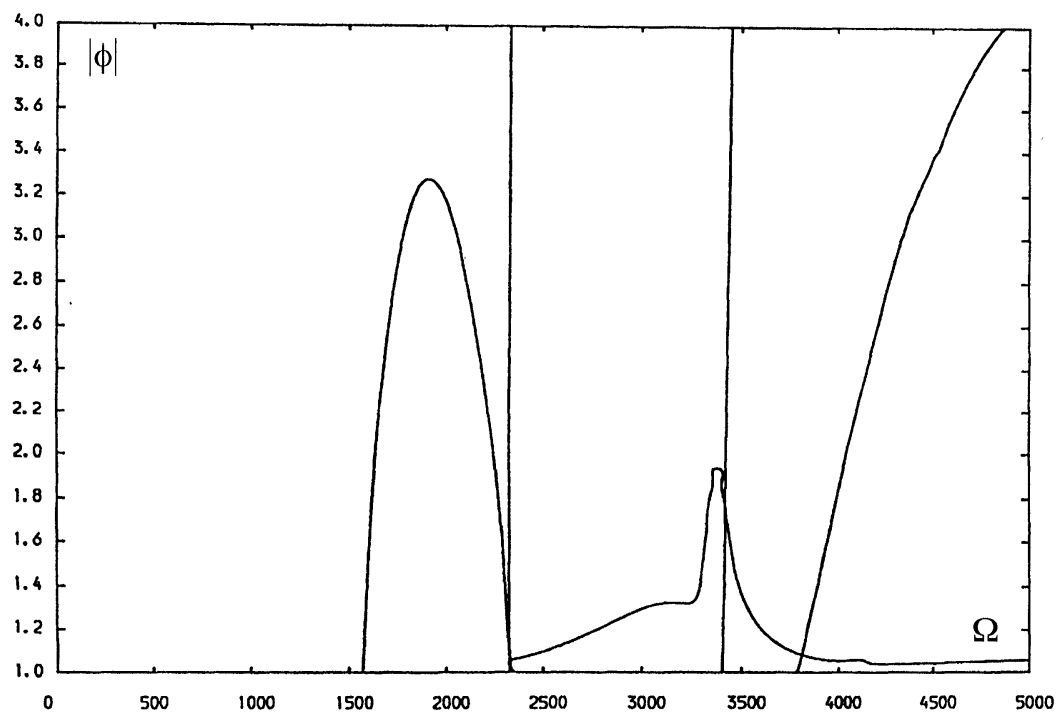

FIGURE 4 (continued) e) 4-pads bearings with oval shaft. f) 4-pads bearings with additional pair of discrete masses.

method applied to pairs of unsymmetric matrices is far from being established in an absolutely reliable manner. Consequently, further activities will concentrate on this subject.

\section{Acknowledgement}

The results presented in this paper are based upon a research project which has been supported by the German Research Foundation-DFG.

\section{NOTATIONS}

$\boldsymbol{A}$ local rotating basis

$\boldsymbol{a}_{i} \quad$ axes of local basis

$c_{\eta}, c_{\zeta}$ stiffness of elastic shaft

$D^{\alpha} \quad$ matrices caused by damping. $\alpha=0 ; 1$

$E \quad$ Young's modulus

$\boldsymbol{E} \quad$ elasticity matrix

$\tilde{\boldsymbol{E}}$ modified elasticity matrix

$\boldsymbol{F} \quad$ Floquet transition matrix

$G \quad$ shear modulus 
$\boldsymbol{G}_{\alpha} \quad$ skew symmetric arrangement of identity vector $\overrightarrow{\boldsymbol{i}}_{\alpha}$

$\overrightarrow{\boldsymbol{i}}_{\alpha} \quad$ identity vector; $\alpha=1,2,3$

$\boldsymbol{K} \quad$ stiffness matrix

$\boldsymbol{M}_{\alpha} \quad$ matrices caused by inertia mass

$\boldsymbol{p} \quad$ column of state variables

$q \quad$ column of degrees of freedom

$\boldsymbol{q}^{+} \quad$ linear variations of $\boldsymbol{q}$

$\boldsymbol{q}_{0} \quad$ quasi static solution

$R \quad$ radius of cylindrical shell

$T \quad$ time period $T=2 \pi / \Omega$

$t$ time, thickness of shell

$\boldsymbol{u} \quad$ column of displacements

$u, v, w$ local displacements

$U$ elastic work

$W \quad$ work

$\overrightarrow{\boldsymbol{x}} \quad$ position vector

$\boldsymbol{Y}$ modal basis of complete auxiliary eigenvalue problem

$\boldsymbol{Z} \quad$ modal basis of reduced auxiliary eigenvalue problem

$\beta \quad$ column of local slopes

$\gamma \quad$ column of shear strains

$\delta() \quad$ virtual quantity of ()

$\epsilon \quad$ strains in general

$\boldsymbol{\epsilon}$ column of membrane strains

$\tilde{\boldsymbol{\epsilon}}$ modified column of membrane strains

$\xi, \eta, \zeta$ local coordinates

$\kappa \quad$ column of curvatures

$\lambda$ characteristic argument of Hill's solution

$v \quad$ Poisson's ratio

$\rho \quad$ mass density (mass/volume)

$\phi \quad$ eigenvalues of transition matrix

$\Omega \quad$ angular velocity of rotor

( ) $\times($ ) vector product

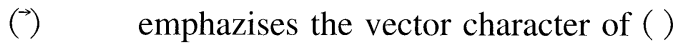

( )* constant part of () with respect to time

\section{References}

Chen, J. and Bogy, D. B., 1992. Natural frequencies and stability of a flexible spinning disk-Stationary load system with rigidbody tilting, J. Applied Mechanics, Vol. 60, pp. 470-477.

Clemens, H. and Wauer, J., 1985. Ebene Eigenschwingungen radial vorbelasteter Kreisringe, ZAMM, Vol. 65, pp. T40-T43.

Endo, M. et al., 1984. Flexural vibration of a thin rotating ring, $J$. Sound Vibration, Vol. 92, pp. 261-272.

Huang, S. and Hsu, B., 1992. Vibration of spinning ring-stiffened thin cylindrical shells, AIAA Journal, Vol. 30, pp. 2291-2298.

Kebari, H. and Cassell, A. C., 1991. Stabilization by non-conforming modes: 9-node membrane element with drilling freedom, Int. J. Num. Meth. Engng., Vol. 31, pp. 1453-1468.

Kebari, H. and Cassell, A. C., 1992. A stabilized 9-node nonlinear shell element, Int. J. Num. Meth. Engng., Vol. 35, pp. 37-61.

Omprakash, V. and Ramamurti, V., 1990. Coupled Free Vibration Characteristics Of Rotating Tuned Blade Disk Systems, J. Sound Vibration, Vol. 140, pp. 413-435.

Ruge, P. and Plonski, T., 1992. Stability of rotating cylindrical shells including nonlinear stiffening, in Rotating Machinery Dynamics, Proc. ISROMAC-3, ed. J. H. Kim, W.-J. Yang, pp. 435443, Hemisphere, Washington.

Sakata, M. et al., 1989. Vibration of bladed flexible rotor due to gyroscopic moment, J. Sound and Vibration, Vol. 131, pp. 417430 .

Seemann, W., 1992. Wave propagation in rotating cylinders, Proc ISROMAC-4, Honolulu, pp. 562-570.

Senker, P., 1993. Stabilitätsanalyse elastischer Rotorsysteme, Braunschweig Series on Mechanics 9-1993, Braunschweig.

Staudinger, G., 1993. Eigenschwingungen eines hochtourigen Rotors mit deformierbarem Scheibenläufer, ZAMM, Vol. 73, pp. T244-T247.

Steigleder, K. and Krämer, E., 1989. Coupled vibration of steam turbine blades and rotors due to torsional excitation by negative sequence currents, Proc. American Power Conf., Chicago, Illinois, pp. 94-102.

Suzuki, K. et al., 1991. Analysis Of Vibrations Of Rotating Thin Circular Cylindrical Shells, JSME Int. J. Series III, Vol. 34, pp. 19-25.

Wauer, J. and Seemann, W., 1989. Eigenfrequenzen rotierender Scheiben unter Berücksichtigung der Grundverformungen, ZAMM, Vol. 69, pp. T339-T341.

Wauer, J., 1977. Stabilität dünner rotierender Kreisringe unter radialem Druck, ZAMM, Vol. 67, pp. T159-T161.

Wittenburg, J., 1977. Dynamics of Systems of Rigid Bodies, Teubner, Stuttgart.

Yano, S. and Kotera, T., 1991. Instability Of The Vibrations Of A Rotating Thin Disk Due To An Additional Support, Archive Of Applied Mechanics, Vol. 61, pp. 110-118. 


\section{ait \\ ENERGY MATERIALS}

M A N E Y publishing

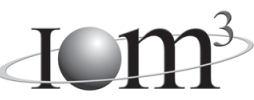

\section{Materials Science \& Engineering for Energy Systems}

Maney Publishing on behalf of the Institute of Materials, Minerals and Mining

The Institute of Materials, Minerals \& Mining

Economic and environmental factors are creating ever greater pressures for the efficient generation, transmission and use of energy. Materials developments are crucial to progress in all these areas: to innovation in design; to extending lifetime and maintenance intervals; and to successful operation in more demanding environments. Drawing together the broad community with interests in these areas, Energy Materials addresses materials needs in future energy generation, transmission, utilisation, conservation and storage. The journal covers thermal generation and gas turbines; renewable power (wind, wave, tidal, hydro, solar and geothermal); fuel cells (low and high temperature); materials issues relevant to biomass and biotechnology; nuclear power generation (fission and fusion); hydrogen generation and storage in the context of the 'hydrogen economy'; and the transmission and storage of the energy produced.

As well as publishing high-quality peer-reviewed research, Energy Materials promotes discussion of issues common to all sectors, through commissioned reviews and commentaries. The journal includes coverage of energy economics and policy, and broader social issues, since the political and legislative context influence research and investment decisions.

\section{CALL FOR PAPERS}

Contributions to the journal should be submitted online at http://ema.edmgr.com

To view the Notes for Contributors please visit: www.maney.co.uk/journals/notes/ema

Upon publication in 2006, this journal will be available via the Ingenta Connect journals service. To view free sample content online visit: www.ingentaconnect.com/content/maney

For further information please contact:

Maney Publishing UK

Tel: +44 (0)113 2497481 Fax: +44 (0)1132486983 Email: subscriptions@maney.co.uk

or

Maney Publishing North America

Tel (toll free): 8662975154 Fax: 6173546875 Email: maney@maneyusa.com

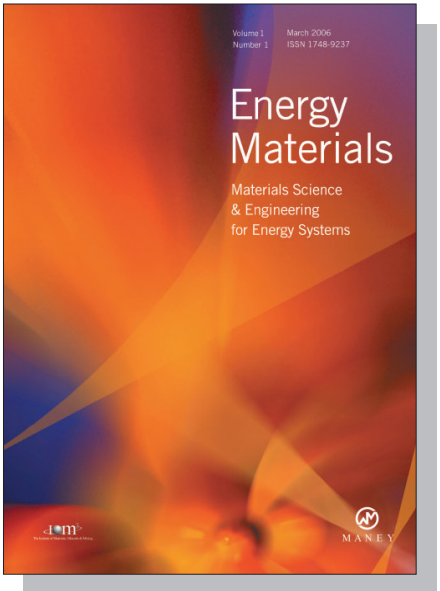

EDITORS

Dr Fujio Abe

NIMS, Japan

Dr John Hald, IPL-MPT, Technical University of Denmark, Denmark

Dr R Viswanathan, EPRI, USA

\section{SUBSCRIPTION INFORMATION}

Volume 1 (2006), 4 issues per year

Print ISSN: 1748-9237 Online ISSN: 1748-9245

Individual rate: $£ 76.00 / U S \$ 141.00$

Institutional rate: $£ 235.00 /$ US $\$ 435.00$

Online-only institutional rate: $£ 199.00 / U S \$ 367.00$

For special $\mathrm{IOM}^{3}$ member rates please email

subscriptions@maney.co.uk

\section{For further information or to subscribe online please visit www.maney.co.uk}



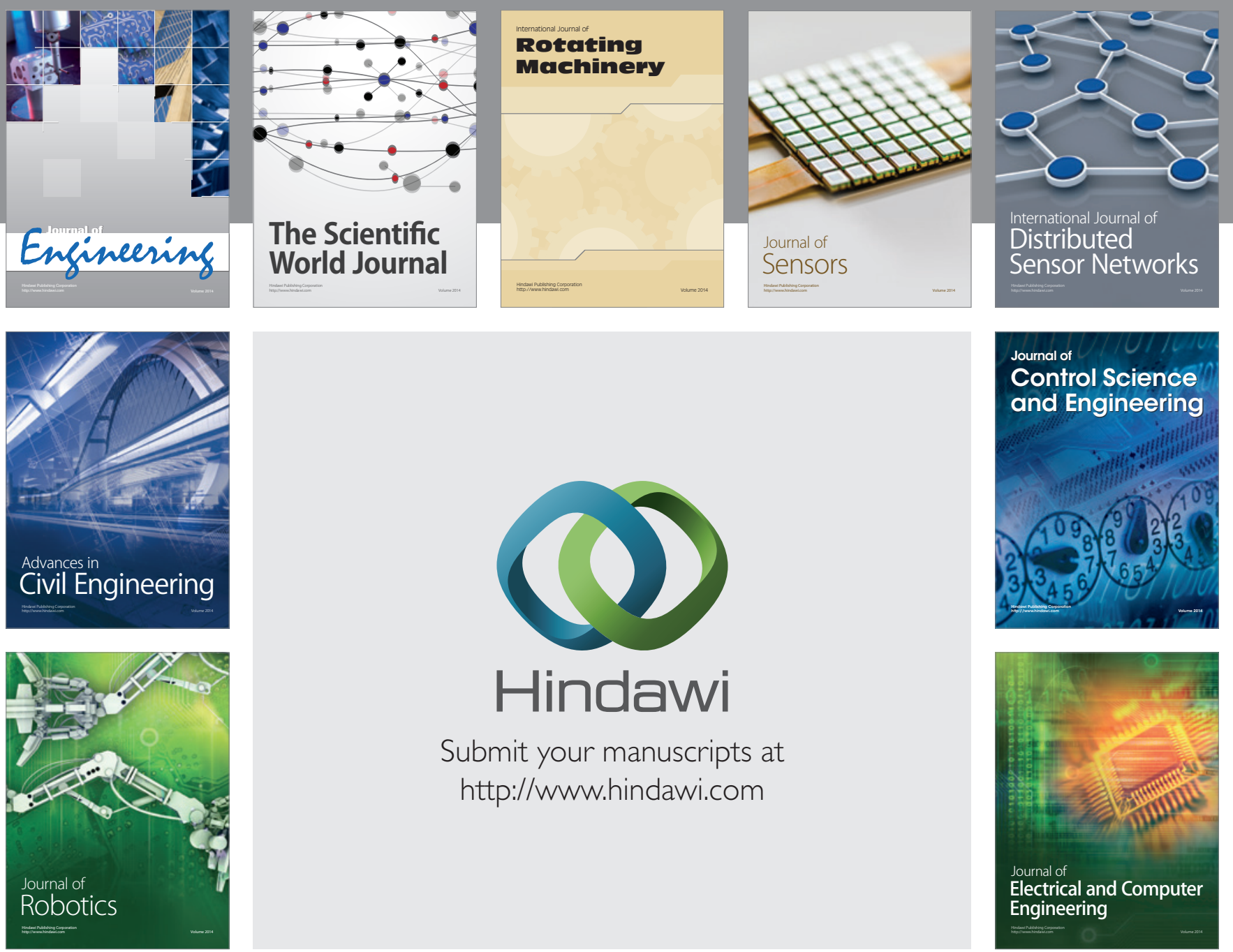

Submit your manuscripts at

http://www.hindawi.com
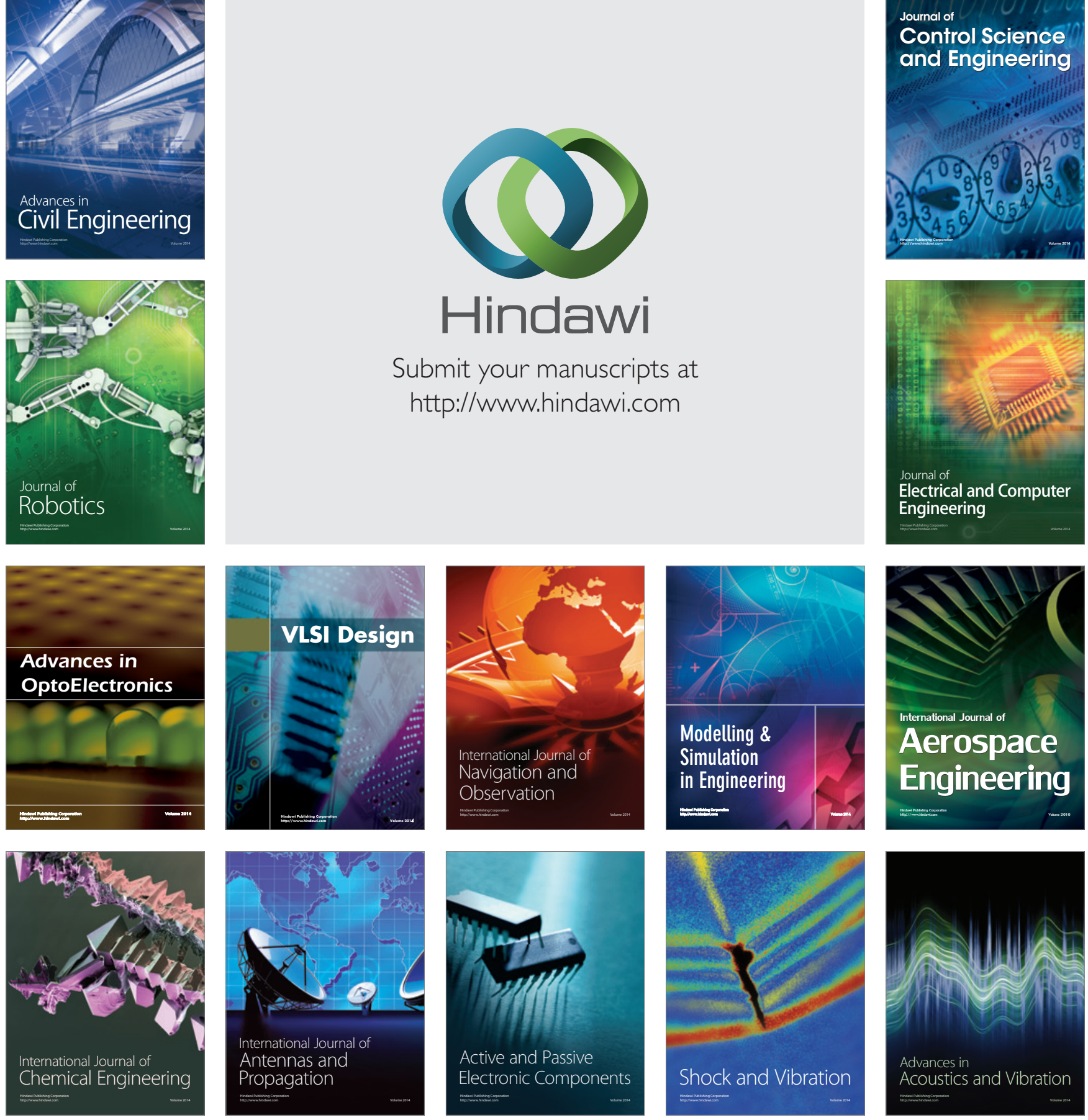